\title{
To Study the Maternal and Neonatal Outcomes of Pregnancies complicated by Rheumatic Heart Disease
}

Uma Pandey

\begin{abstract}
Objectives: To study the maternal and neonatal outcomes of pregnancies complicated by rheumatic heart disease (RHD).

Materials and methods: A retrospective study was carried out in the Obstetric and Cardiology Outpatients Department of Sir Sunder Lal Hospital, Institute of Medical Sciences, Banaras Hindu University, Varanasi, India over a period of 1 year (Dec 2011-2012) involving 96 pregnant patients with rheumatic heart disease. Their maternal and fetal outcomes were reviewed.
\end{abstract}

Results: A total of 96 pregnant mothers with heart disease presented to us during the period of 1 year (Dec 2011-2012). Majority of the pregnant mothers had mitral stenosis $n=46(47 \%)$. Rest of the patients had multivalve disease. Pulmonary arterial hypertension was found to be in 28 patients (29\%). Atrial thrombus was seen in the echocardiography of one patient. Mitral valve replacement was done before pregnancy in 16 mothers (16\%); these gravid mothers were put on low-molecular weight hepanin (LMWH) and during the midtrimester on the Acitrom. There was one patient admitted with atrial fibrillation. New York Heart Association (NYHA) class I and II heart disease progressed in two patients to class III and IV. There was no maternal or fetal mortality in this study. There were 24 cesarean sections (25\%) done out of which 8 were for intrauterine growth restriction (IUGR) $(32 \%)$, rest were due to maternal reasons. There were 12 preterm deliveries. Four fetuses had intraventricular hemorrhage.

Conclusion: Despite no maternal and neonatal mortality, pregnancy in women with heart disease is associated with significant maternal and neonatal morbidities.

Keywords: Pregnancy, Rheumatic heart disease, Maternal outcome, Fetal outcome, Cardiac complication.

How to cite this article: Pandey $U$. To Study the Maternal and Neonatal Outcomes of Pregnancies complicated by Rheumatic Heart Disease. Int J Infertil Fetal Med 2014;5(3):92-94.

Source of support: Nil

Conflict of interest: None

Date of Received: 19-11-14

Date of Acceptance: $26-11-14$

Date of Publication: September 2014

\section{INTRODUCTION}

The incidence of rheumatic heart disease (RHD) among all cardiac disease is approximately $55.7 \% .{ }^{1}$ Incidence of

\footnotetext{
Assistant Professor

Department of Obstetrics and Gynecology, Institute of Medical Sciences, Banaras Hindu University, Varanasi, Uttar Pradesh, India

Corresponding Author: Uma Pandey, Assistant Professor Department of Obstetrics and Gynecology, Institute of Medical Sciences, Banaras Hindu University, Varanasi, Uttar Pradesh, India Phone: 05426703241, e-mail: uma.pandey2006@yahoo.com
}

RHD is still quite common in this part of India and many women present for the first time during pregnancy with RHD. Care in cardiology and obstetrics has been improving at a faster pace than expected. Therefore, we decided to find out both the fetal and maternal outcome in the current day and age in tertiary care center of North India.

Mitral valve is affected most commonly in cases of RHD. Women with severe mitral stenosis (MS) have significant morbidity during pregnancy. It is also understood that not all the medications prescribed for the women with RHD are safe during pregnancy and needs to be changed as per maternal and fetal condition. Appropriate counselling regarding the prognosis before conception and also during this pregnancy is of paramount importance. Counseling would not only help during this pregnancy but also help decide about the future pregnancy.

Confidential enquires conducted in the United Kingdom in the years 2000 to 2002 reported a rise in the incidence of cardiac disease during pregnancy and its associated morbidities and mortalities. There were a total of 44 deaths from heart disease related to pregnancy reported to the enquiry in 2000 to 2002. This compares with 35 deaths in 1997 to 1999, was an increase. Cardiac disease was the second most common cause of maternal death after psychiatric causes in that triennium and more common than the most frequent direct cause of maternal death, thromboembolism. ${ }^{2}$

\section{MATERIALS AND METHODS}

A retrospective study was carried out in the Obstetric and Cardiothoracic Outpatient Departments of Sir Sunder Lal Hospital, Institute of Medical Sciences, Banaras Hindu University, Varanasi, India over a period of 1 year (Dec 2011-2012). All patients were booked. A total of 96 pregnant mothers with rheumatic heart disease presented to us during the period of 1 year (Dec 2011-2012). All the data were recorded on a proforma. Their maternal and fetal outcomes were reviewed.

Maternal observations were made which included worsening of symptoms, need to change or increase the medication, multiple admissions, postpartum cardiac failure and any obstetric complication. Fetal observations included any congenital malformation, low birth weight 
(LBW), intrauterine growth restriction (IUGR), prematurity and incidence of intrauterine death (IUD).

All patients were followed up throughout pregnancy by the same team. Clinic visits were monthly till 32 weeks then fortnightly till 36 weeks and weekly till delivery. Postnatally, women were seen at the end of the puerperium and followed up according to the severity of lesion by the cardiothoracic team. All patients had Doppler echocardiography and electrocardiogram done at the time of booking of pregnancy.

\section{RESULTS}

Total number of deliveries was 1302 and the number of women with RHD were 96, so the incidence was $13.56 \%$ during Dec 2011 to 2012.

Thirty-six women were in the 21 to 25 years of age (Table1) which is nearly similar to studies reported by Sahoo et al and Bhatla et al. ${ }^{3,4}$

Majority of the pregnant mothers had mitral stenosis ( $\mathrm{n}=46)(47 \%)$. In the study by Sahoo et al, incidence of mitral stenosis was found to be $41.6 \%$. This study is in corroboration with that study. Bhatla et al (2003) found mitral stenosis rate of $38.79 \%$ cases in their study. 3,4

Rest of the patients had multivalve disease. Pulmonary arterial hypertension was found to be in 28 patients (29\%). The incidence of moderate to severe mitral stenosis (mitral valve area below $1.5 \mathrm{~cm}^{2}$ by echocardiography) was $24 \%$. Atrial thrombus was seen in the echocardiography of one patient and she was given appropriate thromboprophylaxis.

Mitral valve replacement (MVR mechanical valves) was done before pregnancy in 16 mothers (16\%); these gravid mothers were put on low-molecular weight heparin (LMWH) and during the second trimester on the Acitrom.

Pregnant women with Mitral Valve Replacement (having mechanical valve) were started on LMWH from Coumadin during the first trimester of pregnancy. For the second trimester, they were again started on Coumadin as it was more convenient to the patient. From 34 weeks onwards they were started again on LMWH for the safety of mother and baby. Postnatally, they were started again on Coumadin.

There was one patient admitted with atrial fibrillation. This patient was started on LMWH during pregnancy to prevent thromboembolic complications.

Table 1: Age distribution $(n=96)$

\begin{tabular}{ll}
\hline Age groups (years) & Number of pregnant women \\
\hline$<20$ & 14 \\
$21-25$ & 36 \\
$26-30$ & 24 \\
$31-35$ & 16 \\
$>35$ & 6 \\
\hline
\end{tabular}

Pregnant mothers were categorized according to NYHA class. There were 73 pregnant women who were NYHA I, 19 NYHA II, 1 NYHA III and 3 NYHA IV. New York Heart Association class I and II heart disease progressed in two patients to class III and IV. This is similar to the study done by Sahoo et al. ${ }^{3}$

There were 24 cesarean sections (25\%) done out of which 8 were for IUGR (32\%), rest were due to maternal reasons (labor induction failure, fetal distress, failure of progress of labor). There were 12 (12.5\%) preterm deliveries, in accordance with Avila et $\mathrm{al}^{1}$ (out of which 4 fetuses had intraventricular hemorrhage) and rest were term deliveries $n=84$ (87.5\%). There were $65(67.70 \%)$ spontaneous vaginal deliveries, 7 (7.29\%) outlet forceps delivery, $24(25 \%)$ cesarean section. Induction of labor was done for obstetric indications only.

There were three pregnant mothers who had congestive cardiac failure. They were admitted in the obstetric unit. They were advised bed rest, salt restricted diet, digoxin and furosemide. They recovered quickly.

All pregnant women were on infective endocarditis prophylaxis before pregnancy. There were no new cases of infective endocarditis, which is in corroboration to the study by Sahoo et al. ${ }^{3}$

Pregnant mothers were booked between 6 weeks to 18 weeks of gestation. There were total of 12 women who had preterm labor and eight of them had of idiopathic origin while in three women it happened as they had congestive cardiac failure and one with atrial fibrillation. Total of 8 women were induced, antepartum hemorrhage $=3$, gestational diabetes mellitus $=3$ and pre-eclamptic toxaemia $=2$. Majority of the women did well in the postpartum period. But, four women had postpartum hemorrhage in the immediate postpartum period while five women were anemic which was present before delivery.

There were lower rates of cardiac complications (Table 2) in this study $6.25 \%$ compared to the other studies which could be explained due to early referral, better education and availability of modern diagnostic gadgets. There was just one case of atrial fibrillation while there was no case of venous thromboembolism. ${ }^{1,4}$

Unfortunately, we do not have the data regarding details of anemia before pregnancy and causes of anemia. We apologize for that.

Maternal obstetrical complication (Table 3) rate was $22.91 \%$ which is in accordance to the studies done by Sahoo et al and Bhatla et al. ${ }^{3,4}$

Intrauterine growth restriction was found to be $8 \%$ (Table 4) while the percentage of neonatal complication was $28 \%$. This is nearly similar to the study done by Sahoo et al and Siu et al. ${ }^{3,5}$ 
Table 2: Maternal cardiac complication

\begin{tabular}{lll}
\hline Cardiac complications & Number & Percentage (\%) \\
\hline Congestive heart failure & 3 & 3.1 \\
Atrial fibrillation & 1 & 1.04 \\
Infective endocarditis & 0 & 0 \\
VTE & 0 & 0 \\
Deterioration in NYHA class & 2 & 2.08 \\
Maternal death & 0 & 0 \\
\hline
\end{tabular}

NYHA: New York Heart Association; VTE: Venous thromboembolism

Table 3: Maternal obstetrical complication

\begin{tabular}{lll}
\hline Obstetrical complication & Number & Percentage (\%) \\
\hline Pregnancy-induced hypertension & 2 & 2.08 \\
Gestational diabetes & 3 & 3.1 \\
Severe anemia & 5 & 5.20 \\
Multiple pregnancy & 0 & 0 \\
Previous cesarean & 5 & 5.20 \\
Antepartum hemorrhage & 3 & 3.1 \\
Postpartum hemorrhage & 4 & 4.1 \\
Abortions & 0 & 0 \\
Maternal death & 0 & 0 \\
\hline
\end{tabular}

Table 4: Fetal complications

\begin{tabular}{lll}
\hline Fetal complications & Number & Percentage (\%) \\
\hline IUD & 3 & 3.12 \\
Preterm & 12 & 12.5 \\
IUGR & 8 & 8.33 \\
Intraventricular hemorrhage & 4 & 4.16 \\
\hline
\end{tabular}

IUD: Intrauterine device; IUGR: Intrauterine growth restriction

\section{DISCUSSION}

Rheumatic heart disease pregnant mothers have relatively better prognosis compared to congenital cyanotic heart disease, arrhythmias or cardiomyopathy. This is due to the fact that these patients are of young age, in sinus rhythm, and have normal myocardial function.

Echocardiography is a good diagnostic tool and is also safe during pregnancy. With routine subacate bacterial endocarditis, prophylaxis incidence of infective endocarditis (IE) has become almost negligible. Routine examination of cardiovascular system should be done in the antenatal care to detect cardiac diseases. 'Although, this was a small series of pregnant patients but there was no maternal or fetal mortality'. In this study, there were no maternal mortality which is similar to the studies done by Bhatla and Siu et al. ${ }^{4,6}$

These 96 pregnant mothers were followed by the same obstetric and cardiothoracic team in a tertiary referral center of North India. The adherence to guidelines led to no mortality and a lower incidence of morbidities in both mother and baby, moreover adherence to the IE prophylaxis 'according to the clinical guideline' resulted in almost $100 \%$ protection. $^{7}$

New York Heart Association class I and II heart disease progressed in 2 patients to class III and IV. Both of these patients had mitral stenosis. These patients had pulmonary congestion and were treated with furosemide and $\beta$-blockers. 'None of the patient needed' ballon mitral valvotomy during pregnancy but they were made aware that it can be offered if they become symptomatic.

Mechanical prostheses were used in these gravid females, therefore, they were given appropriate anticoagulation. The incidence of lower segment cesarean section was $25 \%$. This was due to IUGR, failed induction or fetal distress and not for cardiac reason.

\section{CONCLUSION}

Despite no maternal and neonatal mortality, pregnancy in women with heart disease is associated with significant maternal and neonatal morbidities which strongly correlate with maternal underlying disease. The result of present study highlights the need of preconceptional and antenatal care of women with a multidisciplinary approach (involving obstetricians, cardiothoracic surgeons and neonatologists). This study also concludes that if there is strict prenatal care and early identification of the NYHA grade at the time of booking prognosis of the pregnancy in women with RHD can be improved. Follow-up of patients by the same team helped us better understand the pathophysiology, complications and resultant outcome.

\section{REFERENCES}

1. Avila WS, Rossi EG, Ramires JAF, et al. Pregnancy in patients with heart disease: experience with 1,000 cases. Clin Cardiol 2003;26(3):135-142.

2. de Swiet M, Nelson-Piercy C. Cardiac disease: Chapter 10. Confidential enquiry into maternal and child health, why mothers die 2000-2002, sixth report. RCOG Press at the Royal College of Obstetricians and Gynaecologists, 27 Sussex Place, Regent's Park, London NW1 4RG UK; 2004. p. 140.

3. Sahoo G, Komal. Obstetric outcome in pregnancy with heart disease. Ind J Perinatol Reprod Biol 2012;2(4):12-16.

4. Bhatla N, Lal S, Behera G, et al. Int J Gynaecol Obstet 2003; 82(2):153-159.

5. Siu SC, Sermer M, Colman JM, et al. Prospective multicentric study of pregnancy outcomes in women with heart disease. Circulation 2001;104:515-521.

6. Dajani AS, Tauber K, Ferrieri P, Peter G, Shulman S. Treatment of acute streptococcal pharyngitis and prevention of rheumatic fever: a statement for health professionals. Committee on Rheumatic Fever, Endocarditis and Kawasaki Disease of the Council on Cardiovascular Disease in the Young, the American Heart Association. Pediatrics 1995;96:758-764.

7. Dajani AS, Bisno AL, Chung KJ, Durak DT, Freed M, Gerber MA, Karchmer AW, Millard HD, Rahimtoola S, Shulman ST. Prevention of bacterial endocarditis: recommendation by the American Heart Association. J Am Med Assoc 1990; 264:2919-2922. 